\title{
Anharmonicity-induced phonon broadening in aluminum at high temperatures
}

\author{
Xiaoli Tang, Chen W. Li, and B. Fultz \\ California Institute of Technology, W. M. Keck Laboratory, 138-78, Pasadena, California 91125, USA
}

(Received 19 October 2010; published 3 November 2010)

\begin{abstract}
Thermal phonon broadening in aluminum was studied by theoretical and experimental methods. Using second-order perturbation theory, phonon linewidths from the third-order anharmonicity were calculated from first-principles density-functional theory (DFT) with the supercell finite-displacement method. The importance of all three-phonon processes were assessed and individual phonon broadenings are presented. The good agreement between calculations and prior measurements of phonon linewidths at $300 \mathrm{~K}$ and new measurements of the phonon density of states to $750 \mathrm{~K}$ indicates that the third-order phonon-phonon interactions calculated from DFT can account for the lifetime broadenings of phonons in aluminum to at least $80 \%$ of its melting temperature.
\end{abstract}

DOI: 10.1103/PhysRevB.82.184301

PACS number(s): 63.20.kg, 63.20.dk, 78.70.Nx

\section{INTRODUCTION}

The shortening of phonon lifetimes owing to phononphonon interactions is a measure of lattice anharmonicity. ${ }^{1} \mathrm{It}$ is also closely related to nonequilibrium excitations of phonons and their evolution toward the ground state. ${ }^{2}$ The method for calculating phonon lifetimes by perturbation theory has been known for some time, ${ }^{3}$ but quantitative evaluations require accurate energies and polarizations for all phonons, and accurate anharmonic force constants. Thirdorder anharmonic force constants are typically obtained from simple models consistent with empirical anharmonic elastic constants or thermal expansion. ${ }^{4,5}$ First-principles calculations of phonon linewidths have been performed with frozen phonon methods, ${ }^{6}$ which also allow a relatively easy calculation of phonon energy shifts. ${ }^{7,8}$ More recent work on phonon lifetime broadening has been based on densityfunctional perturbation theory (DFPT). ${ }^{9}$ This approach is often used for optical phonons ${ }^{10-12}$ but only a few firstprinciples calculations have been performed for phonons outside the center of the Brillouin zone (BZ). ${ }^{13,14}$ Recently, Tang and Dong ${ }^{15,16}$ extended the direct supercell finitedisplacement (SCFD) method to calculate the third-order anharmonicity tensor. While DFPT and frozen phonon methods are efficient for studying the anharmonicity of a particular phonon, the SCFD method gives a direct evaluation of force constants, and is more efficient for assessments of all phonons in the BZ. Nevertheless, although ab initio methods can often calculate harmonic phonon dynamics with reasonable accuracy, including the anharmonicity is a greater risk, and it is even less clear if three-phonon processes are sufficient to account for phonon lifetime broadenings at elevated temperatures.

Aluminum, a simple metal well suited for plane-wave density-functional theory (DFT) calculations, is a good test case for calculations of anharmonic effects. Phonon dispersions and linewidths in aluminum were measured by neutron spectrometry ${ }^{17}$ at 80 and $300 \mathrm{~K}$, and a few modes were studied at $800 \mathrm{~K}^{18}$ The phonon density of states (DOS) was measured at temperatures to $775 \mathrm{~K}$, showing a surprisingly large broadening ${ }^{19}$ but small energy shifts that seemed consistent with conventional quasiharmonic behavior from ther- mal expansion. Some theoretical studies ${ }^{20-22}$ assessed the phonon broadening using model potentials. Effects on phonons from the electron-phonon interaction were calculated within DFPT, ${ }^{23}$ but at high temperatures the phononphonon interactions are more important in aluminum. ${ }^{24,25}$

Here we report results from an $a b$ initio study of the effects of three-phonon processes on the lifetimes of all phonons in aluminum. We find excellent agreement with experimental results on linewidths from triple-axis neutronscattering measurements along high-symmetry directions. ${ }^{17}$ We also preformed new measurements on the phonon DOS of aluminum at higher temperatures and the broadening of the neutron spectra are accounted for by the $a b$ initio calculations. This demonstrates, first, that $a b$ initio methods are capable of predicting anharmonic effects, and second, that the anharmonic interactions in aluminum are dominated by third-order phonon-phonon scattering processes to at least $80 \%$ of the melting temperature. The dominant phononphonon scattering processes can then be identified and detailed variations in phonon linewidths with wave vector and temperature are reported.

\section{METHODS}

\section{A. Lattice-dynamical calculations}

The phonon-phonon interaction is treated within secondorder perturbation theory. When only three-phonon scattering processes are considered, the basic scattering processes are annihilation of one phonon with the creation of two other phonons (down conversion), and the annihilation of two phonons with the creation of another (up conversion). Simultaneous annihilations or creations of three phonons are excluded because they violate energy conservation. The phonon linewidth contributed from the leading third-order anharmonicity is ${ }^{3,26}$

$$
\begin{aligned}
2 \Gamma(\vec{q}, j)= & \frac{\pi \hbar}{8 N} \sum_{\vec{q}_{1}, \vec{q}_{2}, j_{1}, j_{2}} \Delta\left(\vec{q}+\vec{q}_{1}+\vec{q}_{2}\right) \frac{\left|\Phi\left(\vec{q} j ; \vec{q}_{1} j_{1} ; \vec{q}_{2} j_{2}\right)\right|^{2}}{\omega\left(\vec{q}_{1} j_{1}\right) \omega\left(\vec{q}_{2} j_{2}\right) \omega(\vec{q} j)} \\
& \times\left[\left(n_{1}+n_{2}+1\right) \delta\left(\omega-\omega_{1}-\omega_{2}\right)\right. \\
& \left.+2\left(n_{1}-n_{2}\right) \delta\left(\omega+\omega_{1}-\omega_{2}\right)\right],
\end{aligned}
$$


where $N$ is the number of unit cells, $n$ is Planck's distribution function, $\omega_{i}$ is the energy of the phonon mode with wave vector $\vec{q}_{i}$ and branch index $j_{i}$, and $\Phi$ is the Fourier transform of the third-order lattice anharmonic tensor. The first term in the braces corresponds to a down-conversion process where the initial phonon of frequency $\omega$ decays into two lowerenergy phonons of $\omega_{1}$ and $\omega_{2}$. The second term corresponds to an up-conversion process where an initial phonon of $\omega$ is scattered by a thermal phonon $\omega_{1}$ into a phonon of higher energy $\left(\omega_{2}\right)$. The $\Delta$ and $\delta$ functions ensure the conservation of crystal momentum and energy, respectively. The sums extend over the first BZ. Full calculations of phonon widths therefore require knowledge of harmonic lattice dynamics and all applicable third-order anharmonicities. Harmonic lattice dynamics gives information on all one-phonon states that are characterized by energies and polarization vectors while the anharmonic lattice tensor couples the one-phonon states and causes transitions between them.

The VASP software package ${ }^{27}$ was used to calculate total energies and forces with Blöchl's projector augmented wave pseudopotential $^{28}$ and a plane-wave basis set within the local-density approximation to DFT. The equilibrium structure was first determined by the fitting energy-volume relationship to the third-order Birch-Murnaghan equation of state. The computed equilibrium lattice constants were approximately $1 \%$ less than the experimental values. ${ }^{29} \mathrm{~A}$ Methfessel-Paxton smearing of $0.8 \mathrm{eV}$ and a plane-wave energy cutoff of $380 \mathrm{eV}$ gave well-converged total energies. Both harmonic and anharmonic lattice dynamical properties were then obtained with the supercell finite-displacement method. ${ }^{15,30}$ Independent force constants for a 108-atom periodic supercell were identified by the crystal symmetry and the irreducible distorted supercell configurations for the force calculations were determined. Both harmonic and third-order anharmonic tensors were constructed based on the atomic forces that were computed for all these irreducible distorted 108-atom supercell configurations within DFT using a $\vec{k}$-point sampling grid of $2 \times 2 \times 2$. Phonon linewidths were finally calculated based on Eq. (1). A $24 \times 24 \times 24 \vec{q}$-point grid was found to give well-converged results and an additional $32 \times 32 \times 32$ interpolation within the prism around each $\vec{q}$-point was used to more accurately account for energy conservation.

\section{B. Inelastic neutron-scattering experiments}

Aluminum metal of $99.99 \%$ purity was formed as cylinders with outer diameter of $1.7 \mathrm{~cm}$ and total wall thickness of $0.06 \mathrm{~cm}$, giving a ratio of multiply to singly scattered neutrons of only $2 \%$. Inelastic neutron-scattering measurements were performed with a time-of-flight Fermi chopper spectrometer, ARCS, at the Spallation Neutron Source at Oak Ridge National Laboratory. The incident neutron energy was $79.5 \mathrm{meV}$ and each measurement included a total of approximately $1.4 \times 10^{6}$ counts. For temperatures of $7,100,200$, and $300 \mathrm{~K}$, the sample was mounted in a closed-cycle helium refrigerator. For temperatures of 300, 450, 600, and $750 \mathrm{~K}$, the sample was mounted in a low background, electrical resistance furnace designed for vacuum operation.

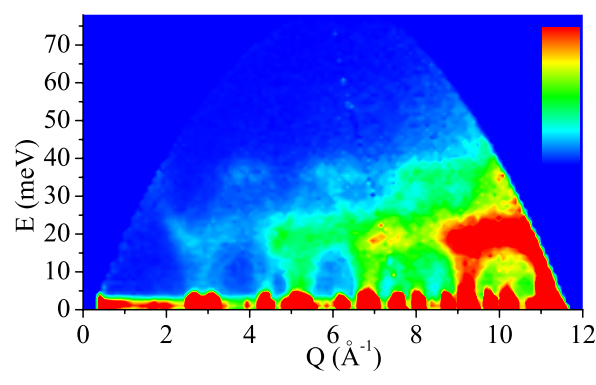

FIG. 1. (Color online) Neutron scattering $I(Q, E)$ from aluminum at $300 \mathrm{~K}$. The vertical axis of the color scale is linear in intensity.

Data reduction was performed with the standard software package for the ARCS instrument. ${ }^{31,32}$ The raw event data of individual neutron detections were first binned to get $I(E, 2 \theta)$, where $2 \theta$ is the scattering angle and $E$ is the energy transfer, and normalized by the proton current on target. Bad detectors were identified and masked, and the data were corrected for detector efficiency using a measurement from vanadium. The $I(E, 2 \theta)$ was then rebinned into intensity, $I(Q, E)$, where $\hbar Q$ is the momentum transfer to the sample. The $Q$ ranged from 0 to $14 \AA^{-1}$, with a bin width of $0.1 \AA^{-1}$, and $E$ ranged from -78.0 to $78.0 \mathrm{meV}$, with a bin width of $1.0 \mathrm{meV}$. The $I(Q, E)$ for $300 \mathrm{~K}$ is shown in Fig. 1. The elastic peak was removed below $8.0 \mathrm{meV}$ and replaced by a function of energy determined from the inelastic scattering just past the elastic peak. ${ }^{19}$ The phonon DOS curves were obtained after corrections for multiphonon and multiple scattering, as described previously. ${ }^{19}$ The averaging over all $Q$ for a given $E$ will eliminate effects of coherent interference between single- and two-phonon scattering. Compared to previous measurements on aluminum, ${ }^{19}$ the increased neutron flux of ARCS made it possible to use thinner samples, which greatly suppressed multiple scattering, but achieved better statistics and energy resolution.

\section{RESULTS AND DISCUSSION}

Figure 2(a) shows the calculated phonon energies along high-symmetry directions with DOS at right. Figure 2(b) shows the correspondent phonon linewidths of these highsymmetry modes. Both phonon energies and linewidths are compared with results from triple-axis neutron-scattering measurements. ${ }^{17}$ The $\vec{q}$-space distribution of phonon linewidths is seen more clearly on four high-symmetry planes in Figs. 2(c)-2(e). The longitudinal-acoustic (LA) phonons are generally much broader than the transverse-acoustic (TA) phonons, and the breadths of the LA phonons vary more strongly with wave vector. The greatest broadening is for LA phonons near square surfaces centered at $\mathrm{X}$ and near hexagonal surfaces centered at $\mathrm{L}$.

The scattering kinematics are given by the two-phonon DOS functions $D_{1}$ and $D_{2}$, which are the BZ sums of second and first products of the $\delta$ and $\Delta$ functions in Eq. (1), ${ }^{33}$

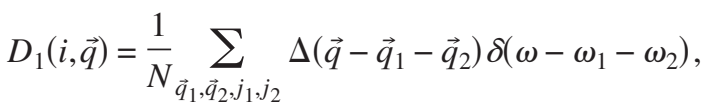



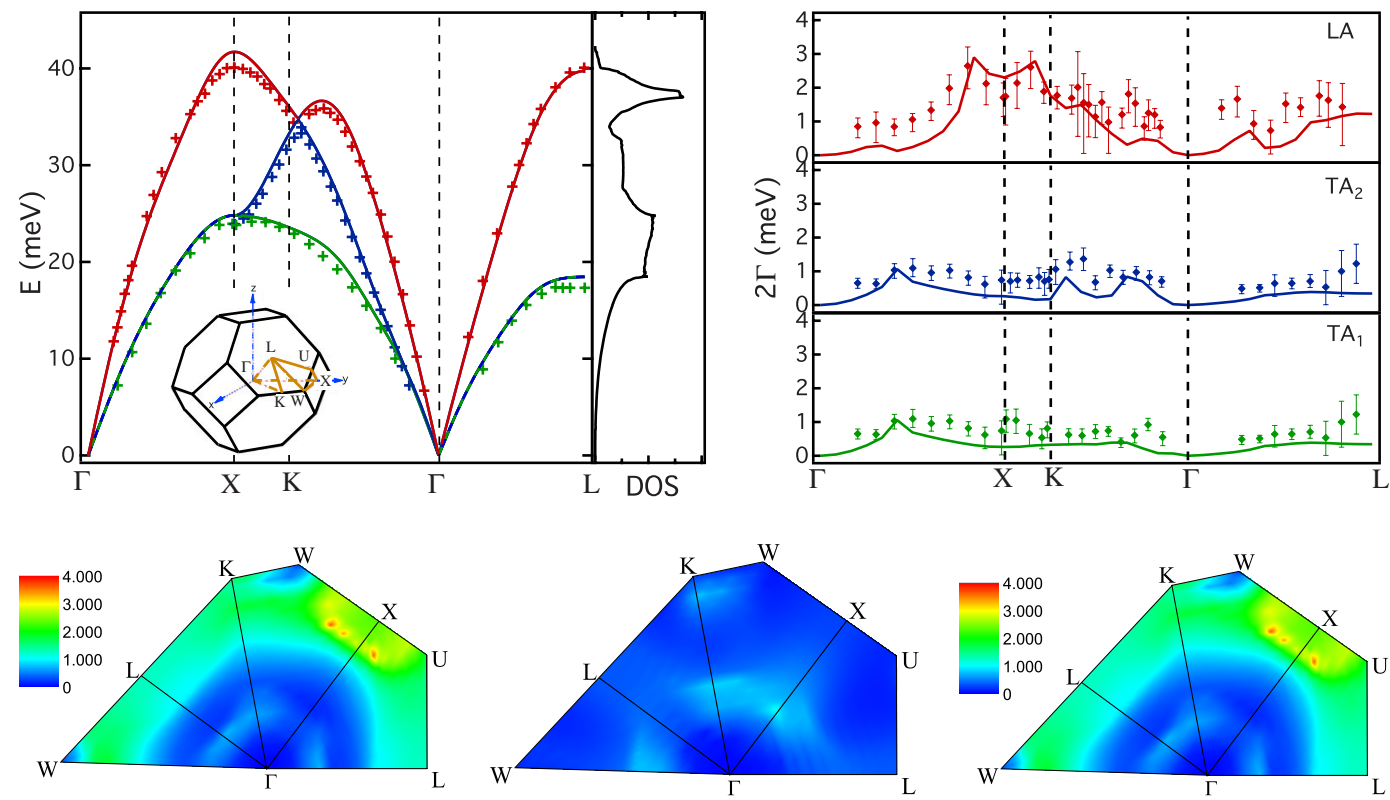

FIG. 2. (Color online) (a) Calculated phonon dispersion at $0 \mathrm{~K}$ with DOS shown at right and (b) correspondent linewidth at $300 \mathrm{~K}$ (solid lines), in comparison with neutron spectroscopy measurement from Ref. 17 (symbols). Linewidth distribution over high-symmetry planes are shown in (c)-(e) for $\mathrm{LA}, \mathrm{TA}_{1}$, and $\mathrm{TA}_{2}$ modes.

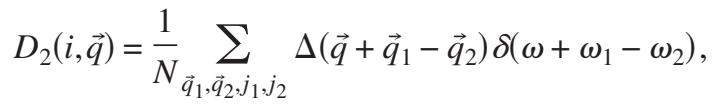

where $D_{1}$ and $D_{2}$ correspond to the down-conversion and up-conversion scattering processes, respectively. Figure 3 shows $D_{1}$ and $D_{2}$ along high-symmetry directions. While TA phonons are mostly scattered by up-conversion processes, LA phonons, especially short-wavelength ones, are mostly scattered by down conversions. The dominant decay channels for LA phonons are LA $\leftrightarrow \mathrm{TA}+\mathrm{TA}(\backsim 85 \%)$ and $\mathrm{LA} \leftrightarrow \mathrm{LA}+\mathrm{TA}(\backsim 15 \%)$. Fewer down-conversion channels become available toward the zone center and up-conversion channels start to open for LA phonons at $\vec{q}$ vectors where

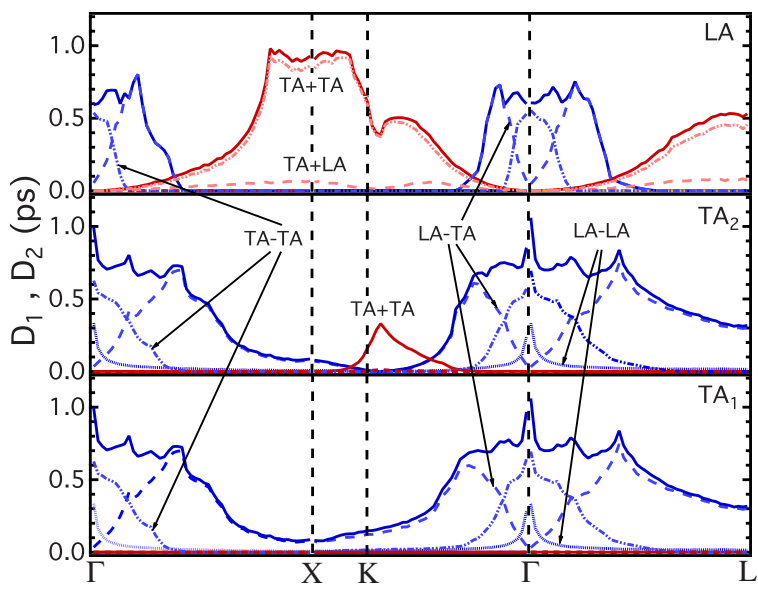

FIG. 3. (Color online) Up-conversion $\left(D_{1}\right)$ processes in blue and down-conversion $\left(D_{2}\right)$ processes in red for three branches along high-symmetry directions. Individual scattering channels are shown by dashed lines. their energies are lower than some TA phonons. The scattering channels change from $\mathrm{LA} \leftrightarrow \mathrm{LA}-\mathrm{TA}$ dominance to LA $\leftrightarrow$ TA-TA dominance when the $\vec{q}$ vector approaches the zone center. Owing to the symmetry of the scattering channels, the dominant scattering channels for transverse phonons are expected to be TA $\leftrightarrow$ LA-TA. Near the zone center, processes such as TA $\leftrightarrow \mathrm{TA}-\mathrm{TA}$ and $\mathrm{TA} \leftrightarrow \mathrm{LA}-\mathrm{LA}$ start to dominate. Large variations in the two-phonon density of states, especially those of LA modes, are clearly responsible for the large variation in phonon linewidth, as one can see by comparing Figs. 2(b) and 3.

Figure 4 shows the phonon linewidths at $300 \mathrm{~K}$ at all irreducible $\vec{q}$ vectors for the different phonon energies. Phonons in the high-energy region have a much larger broadening than in the low-energy region. There is a splitting of the linewidth distribution in the high-energy region, associated with LA phonons near square and hexagonal surfaces of the Brillouin zone [evident from Fig. 2(c)]. These LA phonons are primarily scattered by down conversion into two TA phonons (Fig. 3). The LA phonons with wave vectors near the square surface of the BZ have shorter lifetimes be-

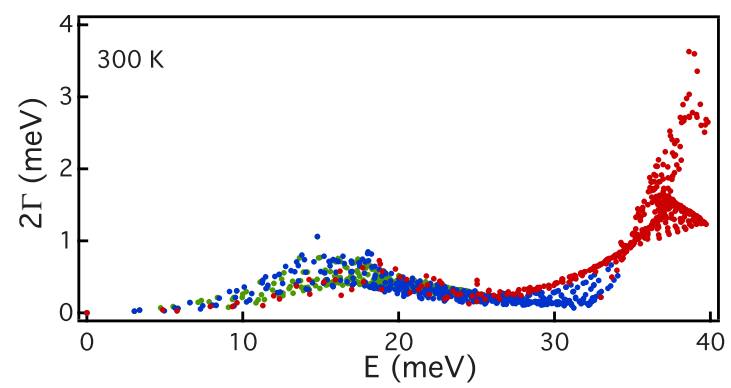

FIG. 4. (Color online) Distribution of phonon broadenings versus phonon energy at $300 \mathrm{~K}$ with $\mathrm{TA}_{1}$ phonons shown in green, $\mathrm{TA}_{2}$ phonons in blue, and LA phonons in red. 


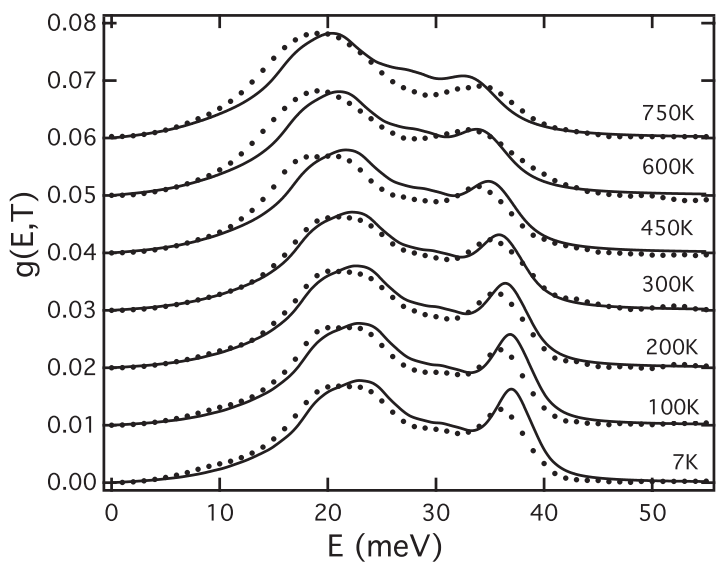

FIG. 5. Comparison of phonon DOS between calculation (solid lines) and inelastic neutron-scattering measurements (points).

cause there are more TA phonons available kinematically for their decay [note the similarity of the LA panels in Figs. 2(b) and 3]. The LA phonons with wave vectors near the square surfaces decay into two TA phonons that are both in the middle energy range where the DOS is high but the LA phonons with wave vectors near hexagonal surfaces can decay into only one TA phonons in the middle energy rangethe other TA phonon falls in the low-energy range near zone center where the DOS is much lower.

To obtain the overall effect of phonon broadening on the phonon DOS, the linewidths of all the phonons at irreducible $\vec{q}$ points on a $24 \times 24 \times 24 \vec{q}$ grid were calculated from 0 to $900 \mathrm{~K}$. Phonon linewidths increase with temperature owing to phonon occupation numbers in Eq. (1) but different phonons broaden with temperature at different rates. Phonon DOS curves at a given temperature, $g(E, T)$, were calculated by considering the phonon broadening from third-order anharmonicity, and the phonon shifts caused by thermal expansion in the quasiharmonic approximation. The energy line shape of each phonon was modeled as a damped harmonic oscillator, ${ }^{34}$

$$
g(E, T)=\int\left\{B\left(E, E^{\prime}, T\right) g\left[E^{\prime}-\Delta E^{\prime}(T)\right] d E^{\prime},\right.
$$

where the damped harmonic oscillator function is

$$
B\left(E, E^{\prime}, T\right)=\frac{2 \Gamma^{\prime}}{\left(\pi E^{\prime} E\right)\left[\left(\frac{E}{E^{\prime}}-\frac{E^{\prime}}{E}\right)^{2}+\left(\frac{2 \Gamma^{\prime}}{E^{\prime}}\right)^{2}\right]}
$$

and the energy shift is

$$
\Delta E^{\prime}=E^{\prime}\left\{\exp \left[-3 \gamma \int_{0}^{T} \alpha\left(T^{\prime}\right) d T^{\prime}\right]-1\right\},
$$

where the mode Grüneisen parameter $\gamma$ was calculated as $d \ln \omega / d \ln V$ and the coefficient of linear thermal expansion $\alpha$ was evaluated with an empirical relation between thermal expansion and heat capacity for aluminum. ${ }^{35}$

Figure 5 compares the calculated and measured phonon
DOS curves. There is a significant broadening of the phonon DOS at higher temperatures and the broader peak around 37 $\mathrm{meV}$ indicates that LA phonons decay faster than TA phonons. The large broadening of these LA phonons gives a prominent tail that extends to higher energies. The overall trend of a larger broadening of LA than TA phonons is in good agreement between computation and experiment. The good agreement between the quasiharmonic phonon shift due to thermal expansion and experiment suggest that the higherorder anharmonicity corrections to the phonon shifts are likely to be small. It is unlikely that the shifts from the thirdorder and fourth-order anharmonicity are canceling for all phonons in the BZ.

The overall agreement between computation and experiment is good but there are discrepancies. Some may be associated with the accuracy of the DFT method for calculating the anharmonicity tensor or from the limitations of perturbation theory when including only third-order phonon-phonon interactions. The lifetime broadening should, in principle, be calculated with phonons for a crystal volume corresponding to each temperature of interest. However, using phonons calculated for an expanded crystal at $600 \mathrm{~K}$ altered the results for most phonon lifetimes by less than $1 \%$, and $5 \%$ for nearzone boundary modes. A major discrepancy comes from the limitations of the analysis of the experimental broadenings when the phonon linewidth is comparable to the energy resolution of the instrument. In principle, the phonon linewidth must approach zero as $q$ goes to zero and this trend is not seen in the experimental data of Fig. 2(b).

\section{CONCLUSIONS}

In summary, the linewidths of all phonons in aluminum were obtained from first-principles density-functional theory with methods using interacting phonon theory in secondorder perturbation theory. The good agreement between the calculated phonon linewidths and previous linewidth measurements at $300 \mathrm{~K}$, and the shapes of the phonon DOS curves to $750 \mathrm{~K}$, not only demonstrate that our method can successfully predict the anharmonicity tensor with DFT, but also indicate that the third-order phonon-phonon interactions are primarily responsible for the lifetime broadening of phonons in aluminum to approximately $80 \%$ of its melting temperature. The most anharmonic phonons in aluminum are found to be those LA phonons near square and hexagonal surfaces of the first Brillouin zone. The major scattering channels for these LA phonons are their decay into two TA phonons. At lower $\vec{q}$, the kinematics favor the up conversion of LA phonons, and the up conversion of TA phonons. Because the phonon dispersions of fcc metals tend to be similar, it seems likely that phonon lifetimes may show similar systematics for other fcc metals, although the $\vec{q}$ of the most anharmonic LA phonons will differ somewhat. We look forward to more extensive applications of these computational methods to account for the effects of phonon-phonon interaction in anharmonic solids, thereby isolating nonharmonic effects from adiabatic electron phonon interactions, for example. 


\section{ACKNOWLEDGMENTS}

This work was supported by DOE BES under Contract No. DE-FG02-03ER46055. The work benefited from software developed in the DANSE project under NSF under
Award No. DMR-0520547. The research at Oak Ridge National Laboratory's Spallation Neutron Source was sponsored by the Scientific User Facilities Division, Office of Basic Energy Sciences, DOE.
${ }^{1}$ N. W. Ashcroft and N. D. Mermin, Solid State Physics (Holt, Rinehart and Winston, New York, 1976), p. 488.

${ }^{2}$ J. A. Kash and J. C. Tsang, in Light Scattering in Solids VI, edited by M. Cardona and G. Güntherodt (Springer, Berlin, 1991), p. 423.

${ }^{3}$ A. A. Maradudin and A. E. Fein, Phys. Rev. 128, 2589 (1962).

${ }^{4}$ R. A. Cowley, J. Phys. (Paris) 26, 659 (1965).

${ }^{5}$ E. Haro, M. Balkanski, R. F. Wallis, and K. H. Wanser, Phys. Rev. B 34, 5358 (1986).

${ }^{6}$ D. Vanderbilt, S. G. Louie, and M. L. Cohen, Phys. Rev. B 33, 8740 (1986).

${ }^{7}$ J. Zarestky, C. Stassis, B. N. Harmon, K.-M. Ho, and C. L. Fu, Phys. Rev. B 28, 697 (1983).

${ }^{8}$ Y.-Y. Ye, Y. Chen, K.-M. Ho, B. N. Harmon, and P.-A. Lindgard, Phys. Rev. Lett. 58, 1769 (1987).

${ }^{9}$ A. Debernardi, S. Baroni, and E. Molinari, Phys. Rev. Lett. 75, 1819 (1995).

${ }^{10}$ G. Lang, K. Karch, M. Schmitt, P. Pavone, A. P. Mayer, R. K. Wehner, and D. Strauch, Phys. Rev. B 59, 6182 (1999).

${ }^{11}$ A. Debernardi, Phys. Rev. B 57, 12847 (1998).

${ }^{12}$ A. Debernardi, C. Ulrich, K. Syassen, and M. Cardona, Phys. Rev. B 59, 6774 (1999)

${ }^{13}$ S. Narasimhan and D. Vanderbilt, Phys. Rev. B 43, 4541 (1991).

${ }^{14}$ G. Deinzer, G. Birner, and D. Strauch, Phys. Rev. B 67, 144304 (2003).

${ }^{15}$ X. Tang and J. Dong, Phys. Earth Planet. Inter. 174, 33 (2009).

${ }^{16}$ X. Tang and J. Dong, Proc. Natl. Acad. Sci. U.S.A. 107, 4539 (2010).

${ }^{17}$ R. Stedman and G. Nilsson, Phys. Rev. 145, 492 (1966).

${ }^{18}$ W. J. L. Buyers, G. Dolling, G. Jacucci, M. L. Klein, and H. R. Glyde, Phys. Rev. B 20, 4859 (1979).
${ }^{19}$ M. Kresch, M. Lucas, O. Delaire, J. Y. Y. Lin, and B. Fultz, Phys. Rev. B 77, 024301 (2008).

${ }^{20}$ T. Högberg and R. Sandström, Phys. Status Solidi B 33, 169 (1969).

${ }^{21}$ T. R. Koehler, N. S. Gillis, and D. C. Wallace, Phys. Rev. B 1, 4521 (1970).

${ }^{22}$ M. Zoli, G. Santoro, V. Bortolani, A. A. Maradudin, and R. F. Wallis, Phys. Rev. B 41, 7507 (1990).

${ }^{23}$ M. M. Dacorogna, M. L. Cohen, and P. K. Lam, Phys. Rev. Lett. 55, 837 (1985).

${ }^{24}$ N. Bock, D. Coffey, and D. C. Wallace, Phys. Rev. B 72, 155120 (2005).

${ }^{25}$ N. Bock, D. C. Wallace, and D. Coffey, Phys. Rev. B 73, 075114 (2006).

${ }^{26}$ R. A. Cowley, Rep. Prog. Phys. 31, 123 (1968).

${ }^{27}$ G. Kresse and J. Furthmüller, Comput. Mater. Sci. 6, 15 (1996);

G. Kresse and J. Hafner, Phys. Rev. B 47, 558 (1993);

G. Kresse and J. Furthmüller, ibid. 54, 11169 (1996).

${ }^{28}$ P. E. Blöchl, Phys. Rev. B 50, 17953 (1994); G. Kresse and D. Joubert, ibid. 59, 1758 (1999).

${ }^{29}$ A. S. Cooper, Acta Crystallogr. 15, 578 (1962).

${ }^{30}$ X. Tang, J. Dong, P. Hutchins, O. Shebanova, J. Gryko, P. Barnes, J. K. Cockcroft, M. Vickers, and P. F. McMillan, Phys. Rev. B 74, 014109 (2006).

${ }^{31} \mathrm{http}: / /$ danse.us/trac/DrChops, 2010.

${ }^{32}$ M. Kresch, O. Delaire, R. Stevens, J. Y. Y. Lin, and B. Fultz, Phys. Rev. B 75, 104301 (2007).

${ }^{33}$ K. Okubo and S. I. Tamura, Phys. Rev. B 28, 4847 (1983).

${ }^{34}$ A. P. French, Vibrations and Waves (Norton, New York, 1971), Chap. 4.

${ }^{35}$ F. C. Nix and D. MacNair, Phys. Rev. 60, 597 (1941). 